\title{
能谱 CT 单能 X 线束成像在脊柱术后中的应用价值
}

\author{
李 强 * 窦文广 梁长华 \\ 新乡医学院第一附属医院，河南 453100
}

\begin{abstract}
摘 要: 目的: 研究能谱 $\mathrm{CT}$ 单能 X 线束成像在脊柱术后中的应用价值。方法: 选取我院自 2018 年 1 月至 2018 年 9 月间已行脊柱内固定术的病人 25 例, 运用能谱扫描模式 (GSI) 进行扫描。将扫描完的图像传送至 GSI 后处理 工作站, 进行单能量重建, 间隔为 $10 \mathrm{keV}$, 将 $40 \mathrm{keV} 140 \mathrm{keV}$ 重建出 11 组单能量的图像。由两名医师分别对 11 组图 像并做一致性检验，用 4 分法对图像进行评分并记录各个单能量时各个分值的人数。结果: 两名医师在对 25 例患者 的 11 组能谱图像分析时, Kappa 值为 0.885 , 即两名医师诊断结果的一致性比较好。6 $60 \mathrm{keV}$ 及以下时, 评分为 3 分及 以上者仅为 1 例 $(1 / 25,4 \%)$; 能量为 $90 \mathrm{keV}$ 时，3 分及以上者为 12 例 $(12 / 25,48 \%)$; 能量为 $100 \mathrm{keV}$ 时, 3 分 及以上者为 23 例 $(23 / 25,92 \%)$; 能量为 $110 \mathrm{keV}$ 时, 3 分及以上者为 21 例 $(21 / 25,84 \%)$; 能量为 $120 \mathrm{keV}$ 时, 3 分及以上者为 16 例 $(16 / 25,64 \%) ; 130 \mathrm{keV}$ 及以上时, 3 分及以上者为 3 例 $(3 / 25,12 \%)$, 图像评分较高、质 量较好主要分布在 $90 \mathrm{keV} \sim 120 \mathrm{keV}$ 能量处。颈椎、胸椎、腰椎在 $100 \mathrm{keV}$ 处的图像质量均最好, 分别为 6 例 $(6 / 7$, $85.71 \%) 、 5$ 例 $(5 / 5,100 \%)$ 和 13 例 $(13 / 13,100 \%)$ 。结论: 能谱 $\mathrm{CT}$ 单能 X 线束成像在脊柱内固定术后的病人 去除伪影有着很大的价值, 在 $100 \mathrm{keV}$ 时图像质量最好, 且在颈椎、胸椎、腰椎没有明显差异。
\end{abstract}

关键词：能谱 $\mathrm{CT}$; 脊柱内固定术; 去除伪影

\section{Application Value of Energy-dispersive CT Single-energy X-ray Beam Imaging in Spinal Surgery}

\author{
Qiang-Li*, Wen-Guang Dou, Chang-Hua Liang \\ First Affiliated Hospital of Xinxiang Medical Colleg, Henan 453100, China
}

\begin{abstract}
Objective: To study the application value of energy-spectrum CT single-energy X-ray beam imaging in spinal surgery. Methods: Twenty-five patients who had undergone spinal internal fixation from January 2018 to September 2018 in our hospital were selected and scanned by energy spectrum scanning mode (GSI). The scanned image is transmitted to the GSI post-processing workstation for single-energy reconstruction at $10 \mathrm{keV}$ intervals. Eleven groups of single-energy images are reconstructed from $40 \mathrm{keV}$ to $140 \mathrm{keV}$. Eleven groups of images were tested by two physicians. The images were scored by four-point method and the number of each score was recorded at each single energy. Results: The Kappa value was 0.885 when the two physicians analyzed 11 groups of energy spectrum images of 25 patients, that is, the consistency of the diagnostic results of the two physicians was better. Only 1 case $(1 / 25,4 \%)$ scored 3 points or more at $60 \mathrm{keV}$ or below; 12 cases $(12 / 25,48 \%)$ scored 3 points or more at $90 \mathrm{keV} ; 23$ cases $(23 / 25,92 \%)$ scored 3 points or more at $100 \mathrm{keV} ; 21$ cases $(21 / 25,84 \%)$ scored 3 points or more at $110 \mathrm{keV}$; and 12 cases $(12 / 25,48 \%)$ scored 3 points or more at $120 \mathrm{keV}$. The former was 16 cases $(16 / 25,64 \%)$ and the latter was 3 cases $(3 / 25,12 \%)$ when $130 \mathrm{keV}$ or above. The images with higher scores and better quality were mainly distributed at $90 \mathrm{keV}$ to $120 \mathrm{keV}$ energy. The image quality of cervical spine, thoracic spine and lumbar spine at $100 \mathrm{keV}$ was the best, with 6 cases $(6 / 7,85.71 \%), 5$ cases $(5 / 5,100 \%)$ and 13 cases $(13 / 13,100 \%)$ respectively. Conclusion: Energy-spectrum CT single-energy X-ray beam imaging has great value in removing artifacts in patients after spinal internal fixation. At $100 \mathrm{keV}$, the image quality is the best, and there is no significant difference in cervical, thoracic and lumbar spine.
\end{abstract}

Keywords: Energy spectrum CT; spinal internal fixation; artifact removal

*通讯作者: 李强, 1981 年 11 月, 男, 汉, 山西武乡人, 主管技师, 学士。研究方向: $\mathrm{CT}_{0}$ 


\section{一、引言}

脊柱结核、脊柱滑脱、脊椎骨折以及脊柱畸形矫正术后常常会使用内固定术来进行治疗, 术后经常会使用影像学 检查手段来对内固定的位置及并发症进行评估, 且术后复查及脊柱的其他病变也会用到无创、快速的影像学检查手 段。常用的影像学检查有经济、快速且辐射剂量比较小的X线检查, 亦有空间分辨率及密度分辨率都较X线有很大提 升但辐射剂量较大的计算机X射线断层扫描技术 ( CT ) 检查, 更有软组织分辨力很高且无电离辐射但价格比较昂贵的 磁共振成像 (MRI) 检查。但是内固定使用的材料多为金属, 这就限制了术后影像学检查方法的选择, 尤其是MRI, 因为体内有金属植人物为MRI的禁忌症。此时, 平常多用于脊柱病变检查的X线及CT的图像效果也是差强人意的。因 为由于金属内固定的植人, 在其周围常常会出现影响图像质量的放射状伪影, 这对于内固定周围组织病变的清晰显示 是极为不利的 ${ }^{[1]}$ 。能谱CT可以瞬时对高能量和低能量的数据进行采集并可进行单能量的重建, 从而达到减轻金属伪影 影响的效果 ${ }^{[2]}$ 。本文旨在通过对能谱CT单能X线束成像在脊柱术后中的应用价值进行研究, 探讨出图像质量最好、伪 影最少的单能量值，以期能使今后脊柱内固定术后CT检查图像质量有所改善，提高脊柱病变诊断正确率。

\section{二、资料与方法}

(一) 临床资料

选取我院自2016年5月至2018年9月间已行脊柱内固定术的病人 25 例, 其中男性18例, 女性7例, 年龄27-65岁, 平 均 $(54.35 \pm 9.83)$ 岁。其中, 颈椎病变7例, 胸椎病变5例, 腰椎病变13例。

纳人标准：（1）所有患者均为脊柱内固定术后;（2）所有患者内固定所用材料相同；（3）患者一般状况较 好，能配合CT检查。

(二) 扫描设备及方法

25名患者均进行能谱CT扫描，扫描设备为美国GE公司的Discovery CT750 HD宝石能谱CT，运用能谱扫描模式 （GSI）。扫描时，先扫描定位像，再根据定位像将范围设定为患者脊柱内固定区域及其上下一个椎体层面，具体 参数如下: 管电压为瞬时切换的 $80 \mathrm{kVp}$ 和 $140 \mathrm{kVp}$, 而管电流为 $600 \mathrm{~mA}$, 矩阵为 $512 \times 512$, 层厚和间隔均为 $5 \mathrm{~mm}$, 螺距为 0.984 , 重建层厚为 $1.25 \mathrm{~mm}$ 。将扫描完的图像传送至 GSI后处理工作站, 进行单能量重建, 间隔为 $10 \mathrm{keV}$, 将 $40 \mathrm{keV} 140 \mathrm{keV}$ 重建出 11 组单能量的图像。

(三) 图像分析

由两个诊断经验丰富的高年资放射科医生采用双盲法对图像伪影情况进行分析，运用评分的方法对图像质量进行 评价。即图像伪影较重, 周围组织结构显示不清, 严重影响诊断效果的记为1分; 图像伪影重, 但周围组织结构尚能 分辨的记为 2 分; 图像几乎无伪影, 周围组织结构可分辨, 对诊断影响不大的记3分; 图像无伪影, 周围组织结构显示 清晰, 图像质量很好的记4分。每位医师评价图像质量时 11 组图像应选取伪影最大层面, 若图像无伪影, 则选取与有 伪影图像层面相同层面。分别记录两名医师的评分结果，并对判读结果进行一致性检验。

(四) 统计学分析

研究采用SPSS 22.0统计分析软件, 计量资料采用 $\mathrm{X} \pm \mathrm{S}$ 的形式表示, 计数资料用百分数表示; 两名诊断医师的一 致性检验使用Kappa检验, Kappa系数为 $0.81 \sim 1$ 时提示两人诊断一致性很好, $0.61 \sim 0.80$ 时提示两人诊断一致性较好, 0.41 0.60时提示中等一致, 而小于 0.40 则一致性均较差; 组间比较采用卡方检验, $P<0.05$ 为差异有统计学意义。

\section{三、结果}

(一) 两名医师诊断结果的一致性检验

两名医师在对 25 例患者的 11 组能谱图像分析时, 对二者结果进行一致性检验, Kappa值为 0.885 , 这表明两名医师 诊断结果的一致性比较好。

(二) 各能量评分结果

图像评分 3 分及以上者认定为图像质量为优, 因为此时的图像是不影响影像判读的。结果如下: 在单能量为 $40 \mathrm{keV}$ 及 $50 \mathrm{keV}$ 时, 评分为 3 分及以上为 0 例, 此时是不利于影像结果的判读的; 能量为 $60 \mathrm{keV}$ 时, 3 分及以上者为 1 例 $(1 / 25$, $4 \%)$; 能量为 $70 \mathrm{keV}$ 时， 3 分及以上者为 3 例 $(3 / 25,12 \%)$; 能量为 $80 \mathrm{keV}$ 时，3分及以上者为 7 例 $(7 / 25,28 \%)$; 能量为 $90 \mathrm{keV}$ 时，3分及以上者为 12 例 $(12 / 25,48 \%)$; 能量为 $100 \mathrm{keV}$ 时, 3 分及以上者为 23 例 $(23 / 25,92 \%)$; 能 量为 $110 \mathrm{keV}$ 时，3分及以上者为 21 例 $(21 / 25,84 \%)$; 能量为 $120 \mathrm{keV}$ 时，3分及以上者为 16 例 $(16 / 25,64 \%)$; 能量 为 $130 \mathrm{keV}$ 时, 3 分及以上者为 2 例 $(2 / 25,8 \%)$; 能量为 $140 \mathrm{keV}$ 时, 3 分及以上者为 1 例 $(1 / 25,4 \%)$, 图像评分较 高, 图像质量较好主要分布在 $90 \mathrm{keV} \sim 120 \mathrm{keV}$ 能量处。（见表1）。 
表1 各单能量的图像评分

\begin{tabular}{|c|c|c|c|c|}
\hline 能量 $(\mathrm{keV})$ & 1分 & 2分 & 3分 & 4分 \\
\hline 40 & 24 & 1 & - & - \\
\hline 50 & 22 & 3 & - & - \\
\hline 60 & 21 & 3 & 1 & 1 \\
\hline 70 & 6 & 16 & 3 & 4 \\
\hline 80 & 4 & 14 & 4 & 8 \\
\hline 90 & 2 & 11 & 4 & 19 \\
\hline 100 & - & 2 & 6 & 6 \\
\hline 110 & - & 4 & 10 & - \\
\hline 120 & 1 & 8 & 2 & \\
\hline 130 & 10 & 13 & & \\
\hline
\end{tabular}

（三）各部位评分质量较好结果

颈椎病变中, 在 $100 \mathrm{keV}$ 处观察到图像3分及以上者为6例 $(6 / 7,85.71 \%)$, 胸椎病变中有 5 例 $(5 / 5,100 \%)$, 腰椎病变中有 13 例 $(13 / 13,100 \%)$ 。可见，不管是在颈椎、胸椎还是腰椎病变中，100keV的图像质量均是最好的。 (见表2）。

表2 各部位评分3分及以上人数

\begin{tabular}{|c|c|c|c|}
\hline 能量 $(\mathrm{keV})$ & 颈椎 & 胸椎 & 腰椎 \\
\hline 90 & 5 & 4 & 12 \\
\hline 100 & 6 & 5 & 13 \\
\hline 110 & 5 & 3 & 11 \\
\hline 120 & 2 & 3 & 9 \\
\hline
\end{tabular}

四、讨论

采用金属植人的内固定术在临床上的应用十分广泛，尤其是在骨折的应用中，脊柱骨折等病变也常常会使用到这 一治疗方法 ${ }^{[3-4]}$ 。但是术后常常会需要对治疗效果即内固定放置的位置以及有无并发症进行评估。此时, 就需要借助 于辅助治疗手段了。准确的早期诊断对于治疗方案的及时调整以及治疗效果都是有着极大帮助的。常用的简便快捷的 诊断方法有 CT、X线和MRI等, 虽然体内有金属植人对于MRI来说是禁忌症, 但是目前有研究 ${ }^{[5]}$ 指出, MRI可以应用 在钛合金内固定中，但是研究太少，可能还不足以完全证实其在临床中的切实可行性。而X线由于本身组织重叠比较 多, 且密度分辨率比较差，在图像的诊断效果上是远远不如 $\mathrm{CT}$ 的。

CT虽然在软组织的诊断效果上较之MRI稍差，但是其对骨质结构的显示却比MRI要好，且价钱较之MRI更便宜。 但是金属内固定的放置, 却极大影响了 CT 的诊断结果，它的放射状伪影常常会使得内固定周围的骨质及软组织显示不 清, 这就极易造成不可避免的误诊和漏诊 ${ }^{[6]}$ 。伪影是在影像检查过程中常常会出现的比较普遍的现象, 主要可以分为 扫描中产生的伪影及扫描机器本身的伪影 ${ }^{[7]}$, 其常常会对图像质量造成不同程度的影响, 伪影较重者, 可能会完全使 整个层面的图像显示不清, 严重影响影像结果的判读。近年来, 随着双能 $\mathrm{CT}$ 及能谱 $\mathrm{CT}$ 的应用, 使这一问题有了极大 的改善。能谱 CT在去除金属应用的中应用也逐渐增多 ${ }^{[8-10]}$, 其在脊柱骨折、脊柱侧弯矫形、髋关节置换等内固定术后 的应用也逐渐广泛起来。

金属伪影的产生是由于金属拥有着比人体组织更高的密度和原子序数, 从而比骨组织和软组织在X线上有着更高 的衰减, 导致金属周围的骨质和软组织显示不清。宝石能谱CT可以实现高、低能值的瞬时采集, 采集完成后还可以进 行单能量重建。此时, 就可以选择金属伪影最少甚至没有的层面对图像进行诊断了。较之普通CT, 能谱CT可以检出 伪影所造成的 $C T$ 值的漂移, 从而达到去除伪影的目的 ${ }^{[1]}$ 。能谱 $\mathrm{CT}$ 除了可以获得不同能量的图像外, 还可以降低患者 的辐射剂量, 使患者的健康有了很大的保障。利用能谱CT的后处理GSI技术, 还可以对扫描的对象进行物质的定量分 析, 从而得出物质的化学组成成分 ${ }^{[12-13]}$ 。

在运用能谱CT对脊柱内固定术后患者进行分析时，可以发现，在图像中的金属伪影主要表现为两种，一种是与 金属长轴相平行的低密度伪影, 其对周围组织结构的影响较小; 还有一种就是分布于金属周围的呈高低混杂密度的伪 影, 这种伪影就是本研究中着重去除的部分。因为其仅仅通过影像科医生的诊断经验是很难做出准确判断的。然而, 
通过能谱CT多个单能量的重建, 就可以实现去除这种伪影的目的 ${ }^{[14]}$ 。在这个过程中, 寻找到使图像质量最少的单能量 值就显得极为重要了, 因为这不仅可以使图像诊断正确率有所提升同时还可以节省工作繁忙的影像科医生的时间, 提 高工作效率。本研究结果显示, 单能量为 $60 \mathrm{keV}$ 及以下时, 图像的伪影普遍较重, 评分 3 分及以上者只有 1 例 $(4 \%)$, 随着能量数的增加, 图像质量也越来越好, $100 \mathrm{keV}$ 时, 评分 3 分及以上者有 23 例 $(92 \%)$, 但能量为 $110 \mathrm{keV}$ 时, 3 分及 以上者为 21 例 $(84 \%)$; 能量为 $120 \mathrm{keV}$ 时, 3 分及以上者为 16 例 $(64 \%)$; 能量为 $130 \mathrm{keV}$ 及以上时, 3 分及以上者为 3 例 $(12 \%)$, 图像评分较高, 图像质量较好主要分布在 $90 \mathrm{keV} \sim 120 \mathrm{keV}$ 能量处, 其中 $100 \mathrm{keV}$ 处图像质量最好。部分国 外文献指出 ${ }^{[15-19]}$, 金属植人物对低能量的X线的衰减系数大，高能量X上，衰减系数则会减小。

本次研究还对单能量值对脊柱各个部位的影响作了分析，研究结果显示，不管是颈椎、胸椎还是腰椎，其在 $100 \mathrm{keV}$ 的图像质量都是最好的, 而在其他能量级时的图像 3 分及 3 分以上人数均比较少, 这就提示, $100 \mathrm{keV}$ 进行脊柱 内固定术后诊断时, 对于各个部位的影响是不大的, 这一结果与国内部分文献一致 ${ }^{[20]}$ 。当然, 本研究尚且存在一些不 足, 样本病例还比较好, 对于证实本文观点稍显欠缺。

总之, 能谱 $\mathrm{CT}$ 单能X线束成像在脊柱内固定术后的病人去除伪影有着很大的价值，通过能谱 $\mathrm{CT}$ 的运用，可以明 显提高脊柱内固定术后患者的 CT图像质量, 其在 $100 \mathrm{keV}$ 时图像质量最好, 在颈椎、胸椎、腰椎诊断方面没有明显 差异。

\section{参考文献:}

[1]Ohashi K, El-Khoury GY, Bennett DL, et al. Orthopedic hardware complications diagnosed with multi-detector row CT[J]. Radiology, 2005,237(2):570-577.

[2]Silva AC, Morse BG, Hara AK, et al. Dual-energy (spectral) CT: applications in abdominal imaging[J]. Radiographics, 2011,31:1031-1046.

[3]Roy-CamilleR, Saillant G, Mazel C. Internal fixation of thelumbar spine with pedicle screw plating[J]. Clin Orthop RelatRes, 1986,203:7-17.

[4]王洪伟, 等. 附加经伤椎椎弓根螺钉内固定治疗胸、腰椎骨折的临床评价[J].第三军医大学学报, 2010,32(4):376379.

[5]代自伦,黄声丽.去金属伪影序列对胸腰椎体金属植入患者磁共振影像的影响 [ J]. 中国 CT 和 MRI 杂志, 2015,13(6):104-107.

[6]史自锋.能谱成像去伪影技术在CTPA中的临床价值[D].合肥:安徽医科大学, 2014 .

[7]周泽俊，胡永胜，高斌，等.多层螺旋CT图像伪影的分析.中国CT和MRI杂志, 2008,6:163.

[8]钱玲玲, 李真林, 袁元, 等. 双源 CT 单能谱成像减少脊柱金属固定器伪影的研究 [J]. 放射学实践, 2013,28(12): 12161219.

[9]刘东华,乐剑平,梅尧,等.双能量CT扫描对骨关节金属植入物伪影消除的初步研究[J].中国基层医药, 2014,(s1):32-33.

[10]钱波,钱农,荣伟良,等.骨折金属固定术后双能量CT图像质量的初步研究[J].广东医学, 2012,33(2):235-237.

[11]徐立功,牛金亮,吴爽,等.能谱成像技术减除脊柱金属植入物伪影的临床应用价值[J].中国医疗前沿, 2012,7(6): 57-59.

[12]Lv P, Xiao Z L, Chen K, Gao J. Spectral CT in patients with small HCC: investigation of imagequality and diagnostic accuracy[J]. European Radiology, 2012,22(10):2117-2124.

[13]鞠烨,刘爱连, 汪禾青, 鞠烨, 刘爱连, 汪禾青, 张婷, 马春梅, 刘静红, 伟杰・阿南德, 穆罕默德・阿尼尔.能谱曲线鉴别 诊断良恶性浆膜腔积液[J].中国医学影像技术, 2012,28(12):2239-2242.

[14]Komlosi P, Grady D, Smith JS, et al. Evaluation of monoenergeticimaging to reduce metallicinstrumentation artifacts in computedtomography of the cervical spine[J]. Neurosurg Spine, 2015,22:34-38.

[15]Zhang LJ, Wu SY, Niu JB, et al. Dual energy CT angiography for the evaluation of intracranial aneurysms: image equality, radiation dose, and comparsion with 3D rotational digital subtraction angiography[J]. AJR, 2010,194(1):23-30.

[16]Fletcher JG, Takabashi N, Hartman R, et al. Dual-energy and dual-source CT: is there a role in the abdmen and pelvis[J]. Radiol Clin N Am, 2009,47(1):41-57.

[17]Bamberg F, Dierks A, Nikolaou K, et al. Metal artifact reduction by dual energy computed tomography using monoenergetic extrapolation[J]. Eur Radiol, 2011,21:1424-1429.

[18] Prell D, Kyriakou Y, Struffert T, et al. Metal artifact reduction for clipping and coiling in interventional c-arm CT. 
AJNR Am J Neuroradiol, 2010,31:634-639.8.

[19]Zhang LJ, Zhao YE, Wu SY, et al. Pulmonary embolism detection with dual-energy CT: experimental study of dualsource CT in rabbits. Radiology, 2009,252:61-70.

[20]吴建国,贺伟浩, 贾卫民,等.单源 CT 单能谱成像在脊柱金属内固定术后的初步应用 [J]. 中国医学计算机成像杂志, 2016,22(6):542-546. 